\title{
ULTRASONIC CHARACTERIZATION OF MICROSTRUCTURE IN POWDER METAL ALLOY*
}

\author{
B.R. Tittmann, L.A. Ahlberg, and K. Fertig \\ Rockwell International Science Center \\ Thousand Oaks, California 91360
}

The ultrasonic wave propagation characteristics were neasured for $1 N-100$, a powder metallurgy alloy used for aircraft engine components. This material was selected as a model system for testing the feasibility of characterizing the microstructure of a variety of inhomogeneous media including powder metals, ceramics, castings and composites. The data were obtained for a frequency range from about 2 to $20 \mathrm{MHz}$ and were statistically averaged over numerous volume elements of the samples. Micrographical examination provided size and number distributions for grain and pore structure. The results showed that the predominant source for the ultrasonic attenuation and backscatter was a dense $\left(\sim 100 / \mathrm{mm}^{3}\right)$ distribution of small micropores ( $10 \mu \mathrm{m}$ radius). Two samples with different micropore densities were studied in detail to test the feasibility of calculating from observed microstructural paraneters the frequency dependence of the microstructural backscatter in the regime for which the wavelength is much larger than the size of the individual scattering centers. Excellent agreement was found between predicted and observed values so as to demonstrate the feasibility of solving the forward problem. The results suggest a way towards the nondestructive detection and characterization of anomalous distributions of micropores when conventional ultrasonic imaging is difficult. The findings are potentially significant toward the application of the early detection of porosity during the materials fabrication process and after manufacturing of potential sites for stress induced void coalescence leading to crack initiation and subsequent failure.

\section{INTRODUCTION}

The acquisition and interpretation of ultrasonic data for the inference of microstructural information is not new and has been carried out by several researchers in the recent past [ref. 1-7]. The objective of this work is to apply these techniques to the types of powder metallurgy alloys used in the manufacture of aircraft engine disc components. The particular application motivating this work is the development of an approach to the nondestructive detection and characterization of anomalous microstructure when conventional ultrasonic imaging is difficult. Such an approach could be important tracking porosity during the materials process as

\footnotetext{
*This work was sponsored in part by Rockwell International Research and Development Funds and in part by the Center for Advanced Nondestructive Evaluation, operated by the Ames Laboratory, USDOE, for the Defense Advanced Research Projects Agency and the Air Force Wright Aeronautical Laboratories/Materials Laboratory under Contract No. W-7405-ENG-82.
} 
well as during service as a finished part in the early locating of potential sites for crack initiation.

The material chosen for these tests is $\mathrm{IN}-100$, a Ni-base alloy prepared by Pratt \& Whitney Aircraft Co. This material is used in the F-15 and F-16 fighter aircraft engine at a rate of $1000 \mathrm{lb}$ of billet material per engine to manufacture three compressor discs, four turbine discs and two spacers. The following sections discuss the experimental approach, the analytical models and the comparison between theory and experiment.

\section{SAMPLE CHARACTERIZATION}

This section treats the micrographical and ultrasonic characterization of the samples, the determination of bulk microsructure parameters from the 2D optical micrographs and he ultrasonic measurement technique [ref. 8].

\section{Micrographical Characterization}

Suitable samples were prepared from a number of different parent samples supplied by Pratt \& Whitney Co. Selected sample surfaces were polished and examined under the optical microscope. Figure 1 shows an optical micrograph of the grain structure. Figure 2 gives histogram data displaying the grain size distribution for two samples. These data show a uniform grain size of about $2-4 \mu \mathrm{m}$.

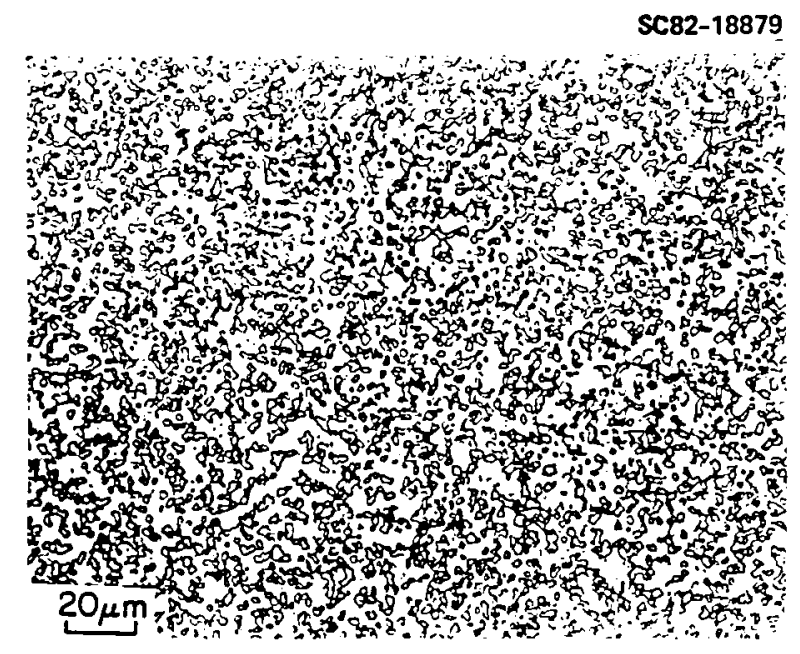

IN 100 GRAIN

Figure 1

Photomicrograph of $\mathrm{IN}-100$ grains.
SC82-18888

\section{GRAIN SIZE IN 100}
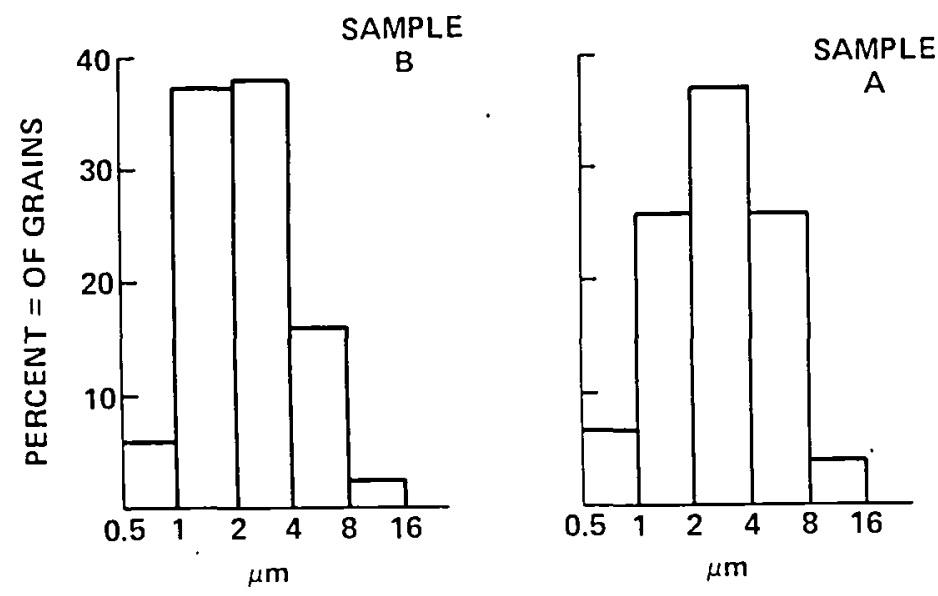

Figure 2

Grain size distribution in IN-100.

One of the samples tested (sample C) is a section of a used F100 engine 2nd disc which was pulled out for spin pit tests. The section is shown in cross-section 
In figure 3 together with lines drawn to indicate traverses with the microscope to study surface features. Figure 4 shows sample microphotos taken along some of these traverses indicating the presence of micropores. The microporosity is a by-product of the powder metallurgy process and has its origin in hollow powder particles with argon gas trapped during the atomization process. Although a sparse, uniform distribution of micropores is not thought to be the limiting factor in low cycle fatigue strength, the micropores do contribute significantly to the ultrasonic noise background and must be taken into account. Table 1 presents a summary of the micropore populations found along the various traverses. Statistical analysis of the micrograph data indicate that the pore distribution is not random but show a tendency towards clustering. Figure 5 presents the information of Table 1 in graphical manner. Figures $6 a$ and $6 \mathrm{~b}$ give pore size distribution histograms for two of the samples tested. Sample A was found to have an approximate micropore density of $330 / \mathrm{mm}^{3}$ whereas sample $C$ had $90 / \mathrm{mm}^{3}$. For sample $C$ the majorlty of micropores fall in the range of between 5 and $10 \mu \mathrm{m}$ in diameter whereas in sample $C$ the diameters range from $\sim 1 \mu \mathrm{m}$ to $50 \mu \mathrm{m}$.

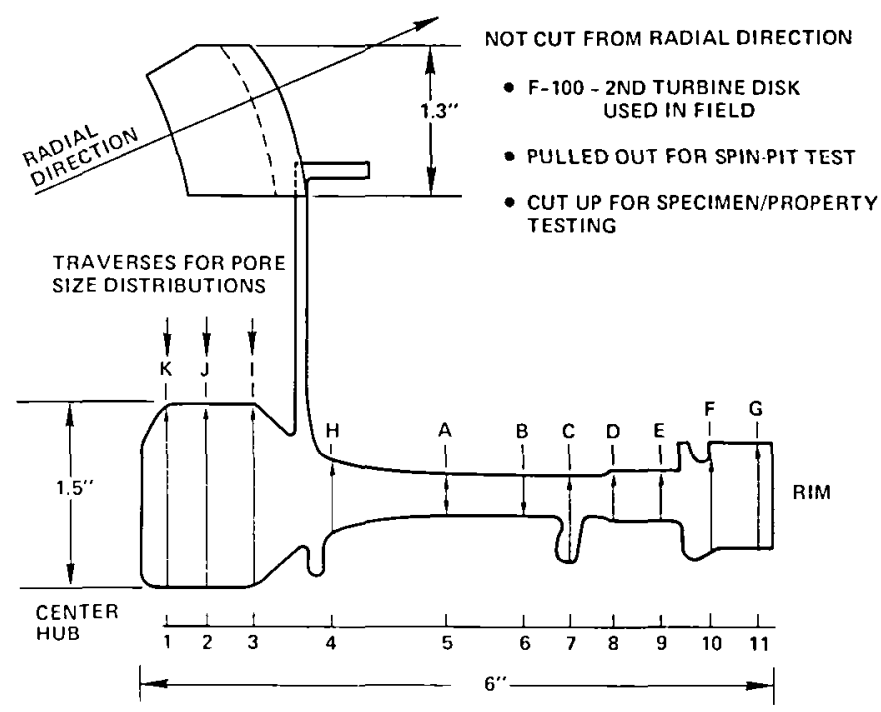

SC82.19519

Figure 3 Section of F100 engine disc.

In order to employ the histogram data obtained from the optical examinations to estimate volume microstructural information, the expressions for the mean volume porosity $\rho_{v}$ and the expectation value for the sixth power of the pore radius $E\left(r^{6}\right)$ were derived:

$$
\begin{aligned}
& \rho_{v}=\frac{1}{\pi} \int_{0}^{\infty} \frac{n_{2}\left(r^{\prime}\right)}{r^{\prime}} d r^{\prime}=\frac{1}{\pi} \sum_{j} \frac{n_{j}}{r_{j}} \Delta r_{j} \\
& E\left(r^{6}\right)=\frac{1}{\rho_{v}} \int_{0}^{\infty} r^{6} n_{3}(r) d r \doteq \frac{8}{\rho_{v}} \sum_{l}\left(\Delta r_{j}\right)\left(r_{j}\right)^{5}\left(n_{j}\right)
\end{aligned}
$$




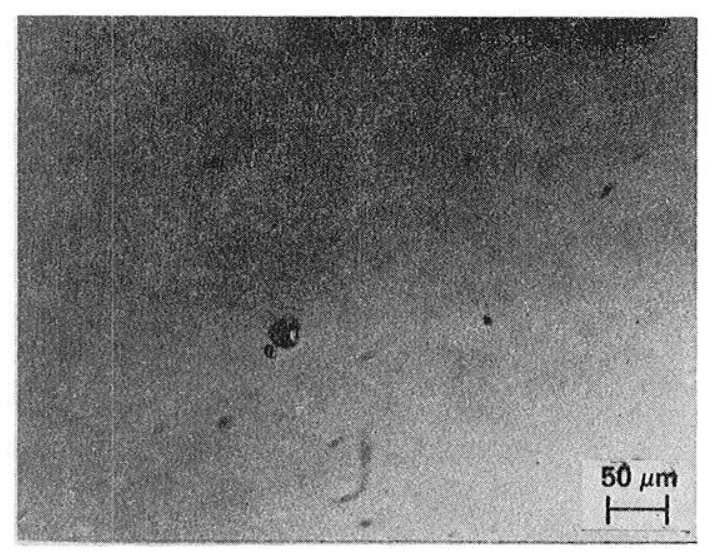

K71

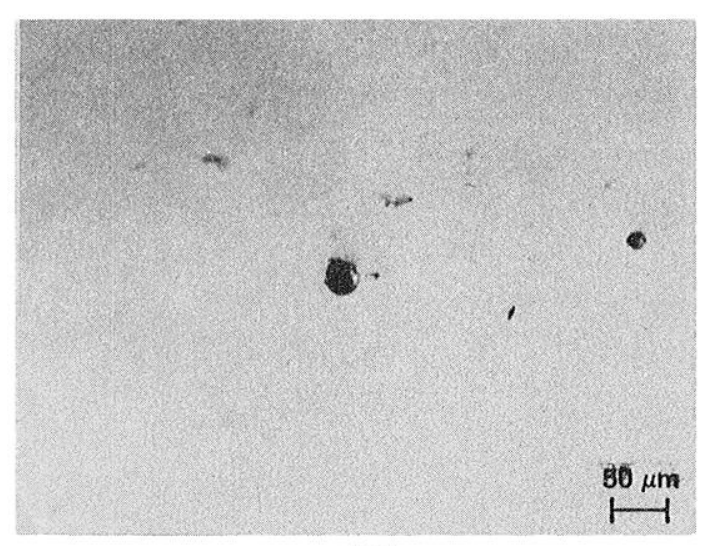

K57

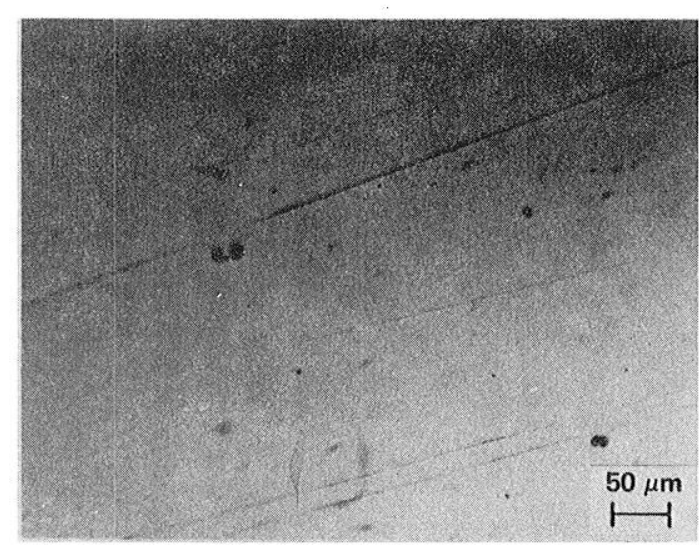

J46

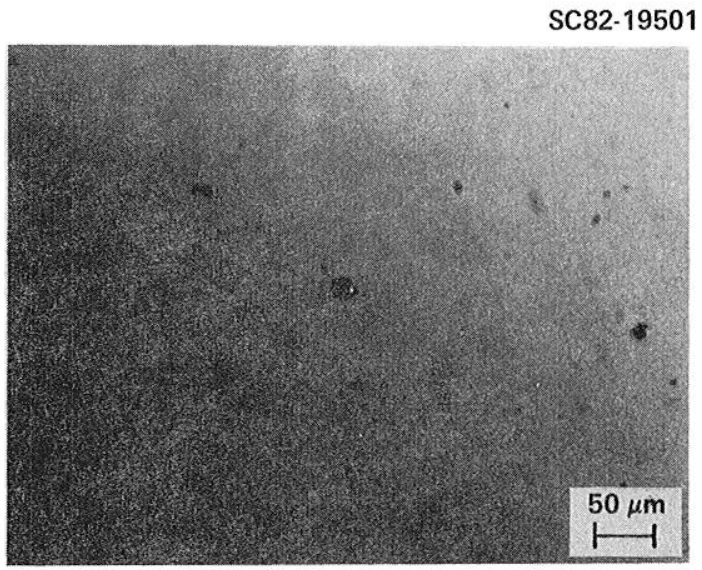

H29

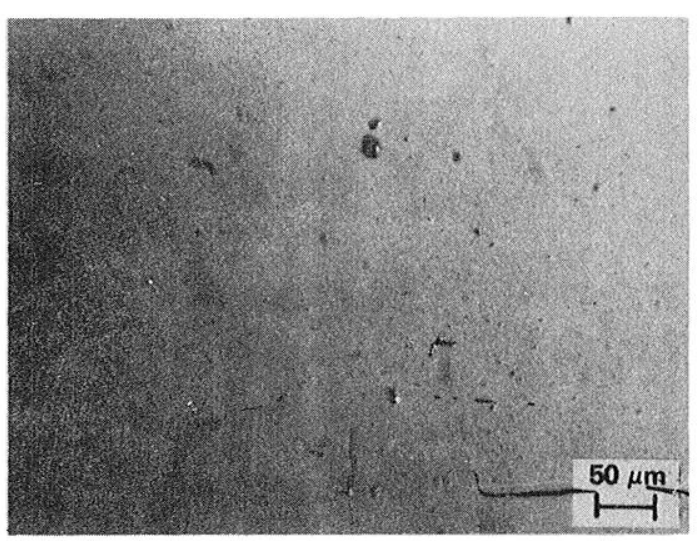

D6

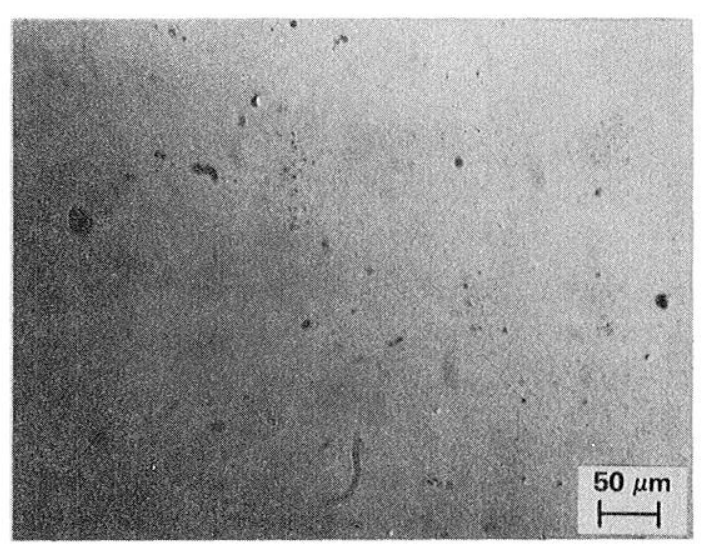

E4

Figure 4 Microphotos of area elements (along tranverses of figure 3) showing micro porosity. 


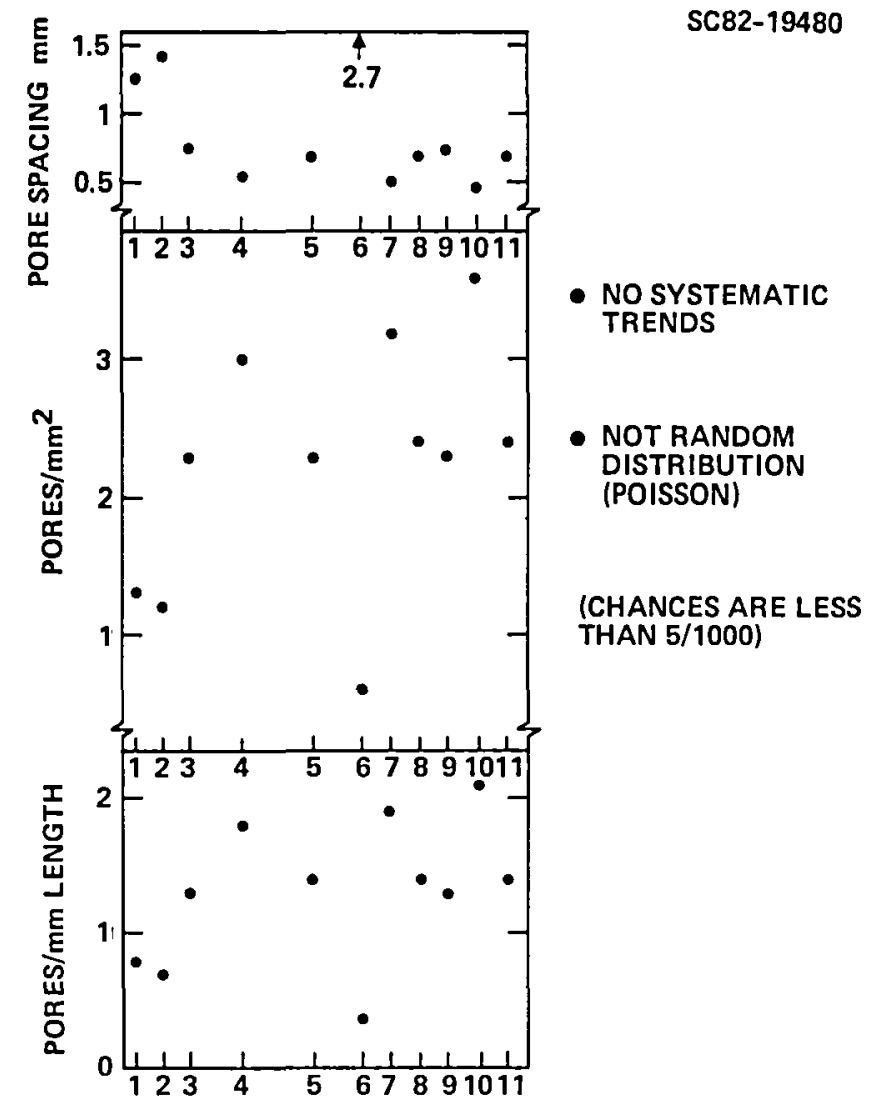

Figure 5 Graph of number of pores per unit length, number of pores per unit area and pore separation plotted vs distance from disc center to run (as indicated by traverse number, see Fig. 3).

SC83-23334
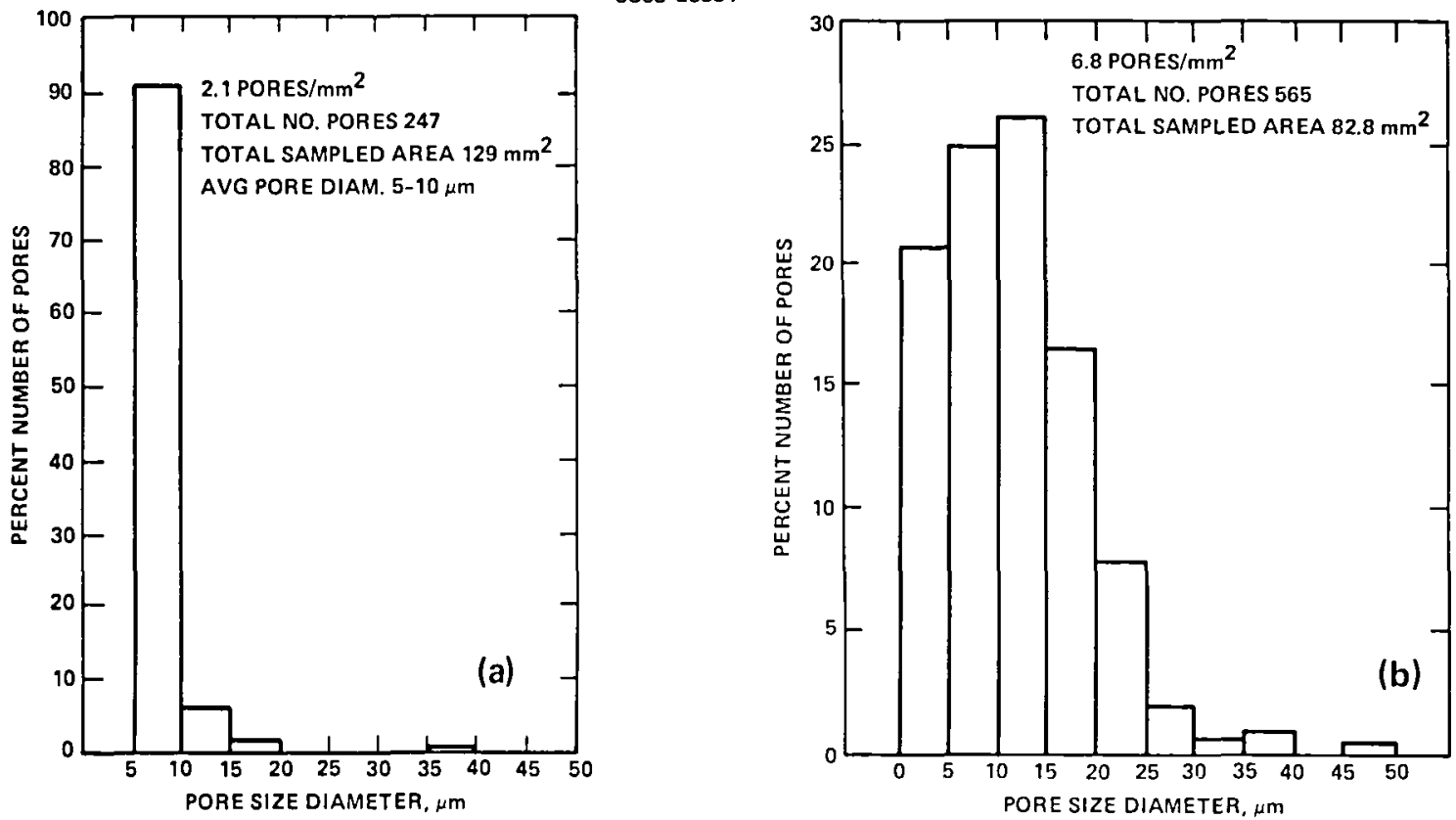

SC83-23338

Figure 6 Pore size distribution (a) sample C - IN-100, (b) sample A - IN-100. 
Table 1. Summary of Observations in Spin Test Sample

\begin{tabular}{|c|c|c|c|c|c|c|c|c|c|}
\hline $\begin{array}{l}\text { Traverse } \\
\text { Number }\end{array}$ & \multicolumn{2}{|c|}{\begin{tabular}{cc}
\multicolumn{2}{c}{ Traverse } \\
Traverse Length \\
Symbol & $\mathrm{mm}$
\end{tabular}} & $\begin{array}{l}\text { Sample } \\
\text { Length } \\
\mathrm{mm}\end{array}$ & $\begin{array}{l}\text { Number } \\
\text { of } \\
\text { Frames }\end{array}$ & $\begin{array}{l}\text { Sample } \\
\text { Area } \\
\left(\mathrm{mm}^{2}\right)\end{array}$ & $\begin{array}{l}\text { Number } \\
\text { of } \\
\text { Pores }\end{array}$ & $\begin{array}{c}\text { Number } \\
\text { of } \\
\text { Pores/ } \\
\text { Linear } \\
\mathrm{mm}\end{array}$ & $\begin{array}{l}\text { Number } \\
\text { of } \\
\text { Pores } \\
\mathrm{mm}^{2}\end{array}$ & $\begin{array}{l}\text { Pore } \\
\text { Spacing } \\
\text { (Av) } m m\end{array}$ \\
\hline 1 & K & 40 & 36.9 & 81 & 21.9 & 29 & 0.79 & 1.3 & 1.27 \\
\hline 2 & $\mathrm{~J}$ & 40 & 39.6 & 87 & 23.5 & 28 & 0.7 & 1.2 & 1.4 \\
\hline 3 & I & 40 & 40.6 & 89 & 24 & 54 & 1.3 & 2.3 & 0.75 \\
\hline 4 & $\mathrm{H}$ & 16 & 15 & 33 & 8.9 & 27 & 1.8 & 3.0 & 0.55 \\
\hline 5 & A & 8 & 7.3 & 16 & 4.3 & 10 & 1.4 & 2.3 & 0.7 \\
\hline 6 & B & 8 & 8.2 & 18 & 4.9 & 3 & 0.36 & 0.6 & 2.7 \\
\hline 7 & c & 19 & 18.2 & 40 & 10.8 & 35 & 1.9 & 3.2 & 0.5 \\
\hline 8 & D & 10 & 10.5 & 23 & 6.2 & 15 & 1.4 & 2.4 & 0.7 \\
\hline 9 & E & 10 & 10.5 & 23 & 6.2 & 14 & 1.3 & 2.3 & 0.75 \\
\hline 10 & $\mathrm{~F}$ & 21 & 16 & 31 & 9.5 & 34 & 2.1 & 3.6 & 0.47 \\
\hline 11 & G & 21 & 15.5 & 34 & 9.2 & $\underline{22}$ & 1.4 & 2.4 & 0.7 \\
\hline \multicolumn{3}{|c|}{ Total Sample } & 218 & & 129 & 271 & 1.27 & 0.87 & \\
\hline \multicolumn{10}{|c|}{ Pore Size Distribution $(\mu \mathrm{m})$} \\
\hline \multicolumn{3}{|c|}{$5-10$} & $10-15$ & $15-20$ & $20-25$ & $25-30$ & \multicolumn{2}{|c|}{$30-35$} & $35-40$ \\
\hline \multirow{2}{*}{\multicolumn{2}{|c|}{$\begin{array}{l}\text { Number of Pores } \\
\text { Percentage }\end{array}$}} & & 18 & 5 & 0 & 0 & & 0 & 1 \\
\hline & & 1.1 & 6.6 & 1.8 & 0 & 0 & & 0 & 0.4 \\
\hline
\end{tabular}

where $n_{2}\left(r^{\prime}\right) d r^{\prime}$ is the number of circles per unit area (with radil between $r^{\prime}$ and $r^{\prime}$ $+\mathrm{dr}^{\prime}$ as measured from micrographs. Each circle represents a cut through a pore by the polished sample surface. $n_{3}\left(r^{\prime}\right)$ is the number of micropores per unit volume element, $\Delta r_{j}$ is the bin width of the pore radius histogram, $r_{j}$ is the average pore radius for each bin and $n_{j}$ is the number of pores in each bin.

These formulae were used for calculations in the detailed examination of two demonstrative samples discussed below.

\section{U1trasonic Characterization}

The samples were studied in two ways. The ultrasonic attenuation was measured In the frequency range from about 5 to $100 \mathrm{MHz}$ on samples $A$ and $B$ with nominally similar pore size distributions. The shear wave backscattered power was measured in the frequency range $2-20 \mathrm{MHz}$ on samples $\mathrm{C}$ and A described in detail in the text.

In the ultrasonic attenuation measurements the data were obtained in the $10 \mathrm{MHz}$ to $100 \mathrm{MHz}$ range of frequencies in several smaller sub-bands. A low attenuation buffer (Al or fused silica) was typically used to obtain the transducer response and the reflection coefficient of the sample interface. The technique and calculations are illustrated in figure 7 for the case of longitudinal waves. For the case of shear waves, an additional technique was employed for rapid data acquisition and is illustrated in figure 8. Here the buffer is water and the angle of incidence is such that the longitudinal waves in the water produce shear waves in the sample. 

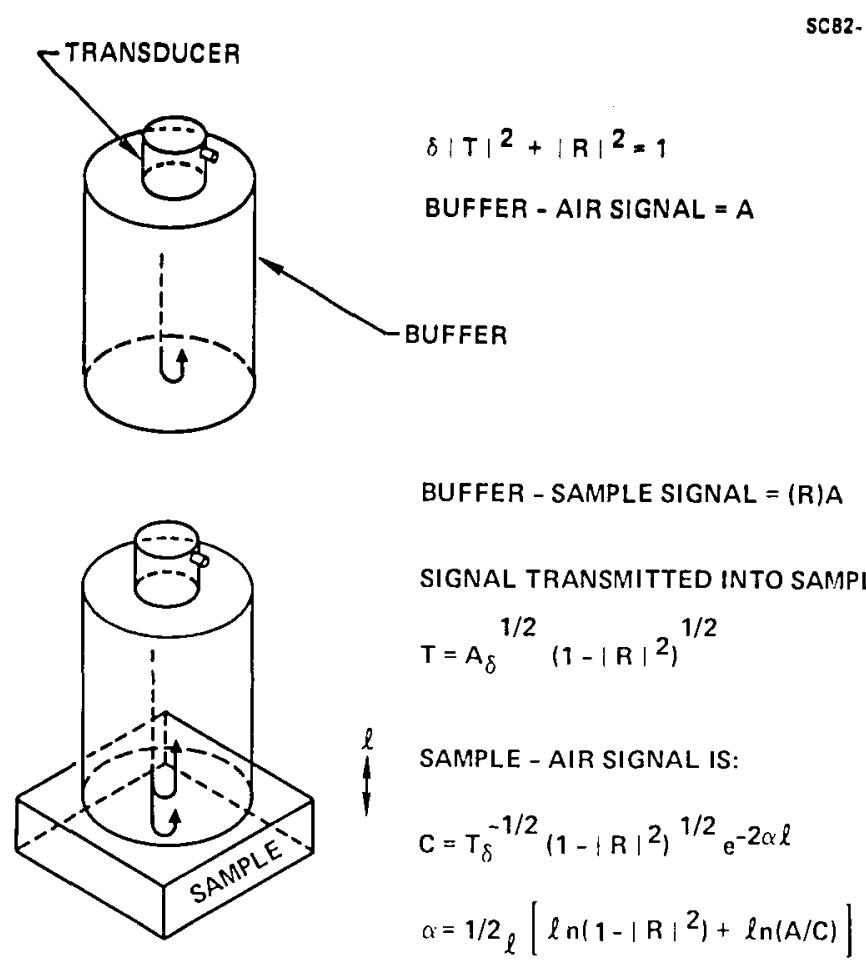

Figure 7 Sample configuration for measurement of longitudinal wave attenuation.

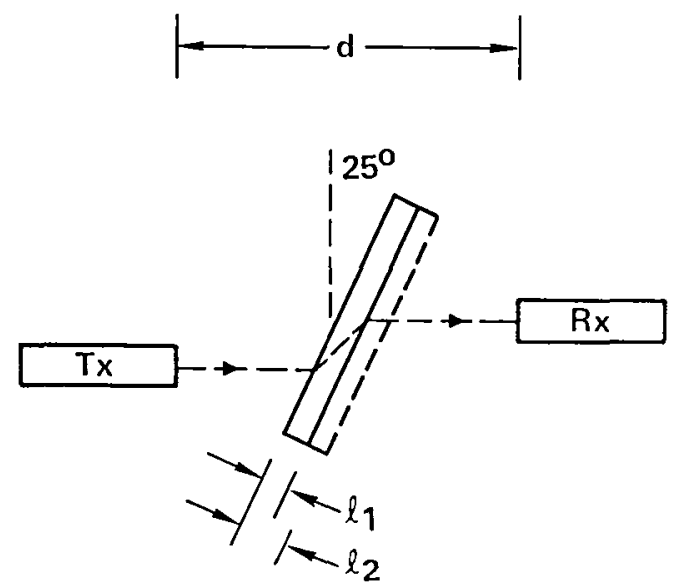

$\operatorname{SC} 82-18891$

$A_{1}=T_{01} T_{10} \exp -\left(\alpha_{s} I_{s 1}+\alpha_{w} I_{w 1}\right)$

$A_{2}=T_{01} T_{10} \exp -\left(\alpha_{s} I_{s 2}+\alpha_{w} I_{w 2}\right)$

$\alpha_{s}=\frac{1}{\Delta l_{s}}\left(\ln \frac{A 1}{A 2}\right)+\alpha_{w}$

Figure 8 Sample configuration for measurement of shear wave attenuation.

Because of the possible generation of leaky waves generated at the interfaces and the non-circular beam spreading associated with non-normal incidence, the measurements were augmented with calibration measurements, carried out with narrow band quartz shear wave transducers in direct tightly bonded contact with the sample. 
In reference to figure $8, \mathrm{~T}_{01}, \mathrm{~T}_{10}$ are the water-sample, sample-water transmission coefficients; $\alpha_{S}$ is the sample shear wave attenuation in nepers $/ \mathrm{cm}, \alpha_{W}$ is the water longitudinal attenuation in nepers/cm, $1_{s 1}, 1_{s 2}$ are the shear wave paths in the sample under test, $1_{w 1}, 1_{w 2}$ are the wave path length in water.

The measurements are summarized in figures 9 and 10 which display attenuation values as a function of frequency for two different samples and for three different locations for the same sample.

$\operatorname{SC} 82-18878$

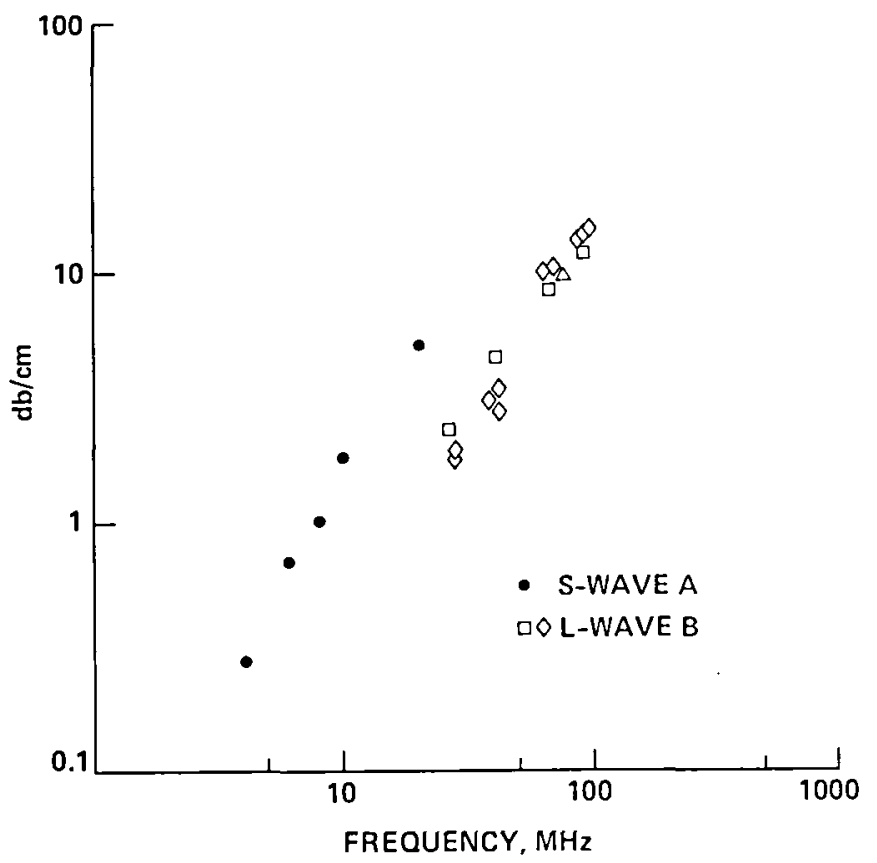

Figure 9

Longitudinal ( $L$ ) and shear (S) wave attenuation for 2 samples of $\mathrm{IN}-100$.

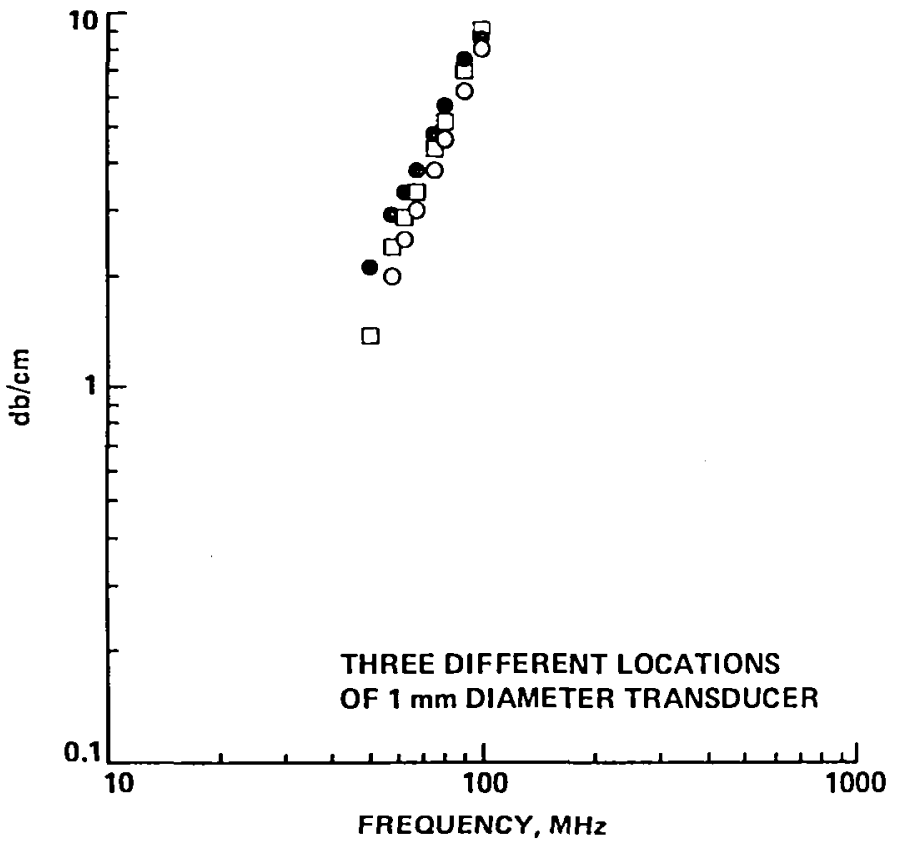

Figure 10

Longltudinal wave attenuation at three different locations on sample $C$.

Table 2 summarizes the information collected by given representative values for attenuation and velocity for two powder metal alloys $\mathrm{IN}-100$ and $\mathrm{T} 1-6246$ compared to a common engine disc material of nonpowder fabrication, 1.e., Waspalloy.

Table 2 .

\begin{tabular}{|c|c|c|c|c|c|c|c|c|}
\hline \multirow[t]{2}{*}{ Sample } & \multicolumn{2}{|c|}{$\begin{array}{l}\text { Velocity } \\
(\mathrm{mm} / \mu \mathrm{m})\end{array}$} & \multirow{2}{*}{$\begin{array}{l}\text { Grain } \\
\text { Size } \\
(\mu \mathrm{m})\end{array}$} & \multicolumn{2}{|c|}{$\begin{array}{l}\text { Atten. } \\
(\mathrm{dB} / \mathrm{cm})\end{array}$} & \multirow[t]{2}{*}{$\begin{array}{l}\text { Freq. } \\
(\mathrm{MHz})\end{array}$} & \multirow{2}{*}{$\begin{array}{c}\text { Freq. } \\
\alpha_{L}\end{array}$} & \multirow{2}{*}{$\begin{array}{l}\text { Dep. } \\
\qquad \alpha_{S}\end{array}$} \\
\hline & $\mathrm{v}_{\mathrm{L}}$ & $\mathrm{v}_{\mathrm{S}}$ & & $\alpha_{L}$ & $\alpha_{S}$ & & & \\
\hline Waspaloy & 6.04 & 3.23 & 100 & 4 & 11 & 10 & $\mathrm{f}^{2.2}$ & $f^{2: 5}$ \\
\hline IN -100 & 6.52 & 3.29 & 2 & $4 \pm 1$ & 20 & 50 & $f^{2}$ & $\mathrm{f}^{2.2}$ \\
\hline $\mathrm{T} 1-6246$ & 6.11 & 3.16 & $2-4$ & 10 & - & 100 & & $\mathrm{f}^{3.5}$ \\
\hline
\end{tabular}




\section{ANALYSIS}

This section presents the analytical developments leading up to the calculations for a comparison between the experimental observations and the predicted frequency dependencles of back-scattering.

The model for the microstructural backscatter (nolse) spectrum we w111 use 18 taken from the work of Fertig and Richardson [ref. 9]. Th1s model accounts for the statistical nature of the scattering process as well as the diffraction assoclated with the measurement system. Consider flgure 11 below. We assume that the time window, $T$, assoclated with the experiment is small enough so that the variation in the sound beam along the z-axis is negliglble within the sampled part. If we consider a scatterer at position $\vec{r} j=\left(\underline{r}_{j}, z_{j}\right)$, with $\underline{r}_{j}=\left(x_{j}, y_{j}\right)$, then the contribution to the Fourier transform of $n(t)$, the measured time dependent wave form, from this scatterer will be

$$
p\left(\omega, \underline{r}_{j} ; z_{1}\right) \exp \left(i \omega \tau_{j}\right) A_{j}(\omega)
$$

where $\tau_{j}=\left(z_{j}-z_{l}\right) /(2 c)$ is the relative time delay of the $j$ th scatterer, $A_{j}(\omega)$ is its scattering amplitude and $p\left(\omega, \underline{r}_{j}\right)$ (suppressing the dependence of $z_{1}$ ) is the system response function.

- SYSTEM RESPONSE FUNCTION FOR DISTRIBUTED SCATTERERS

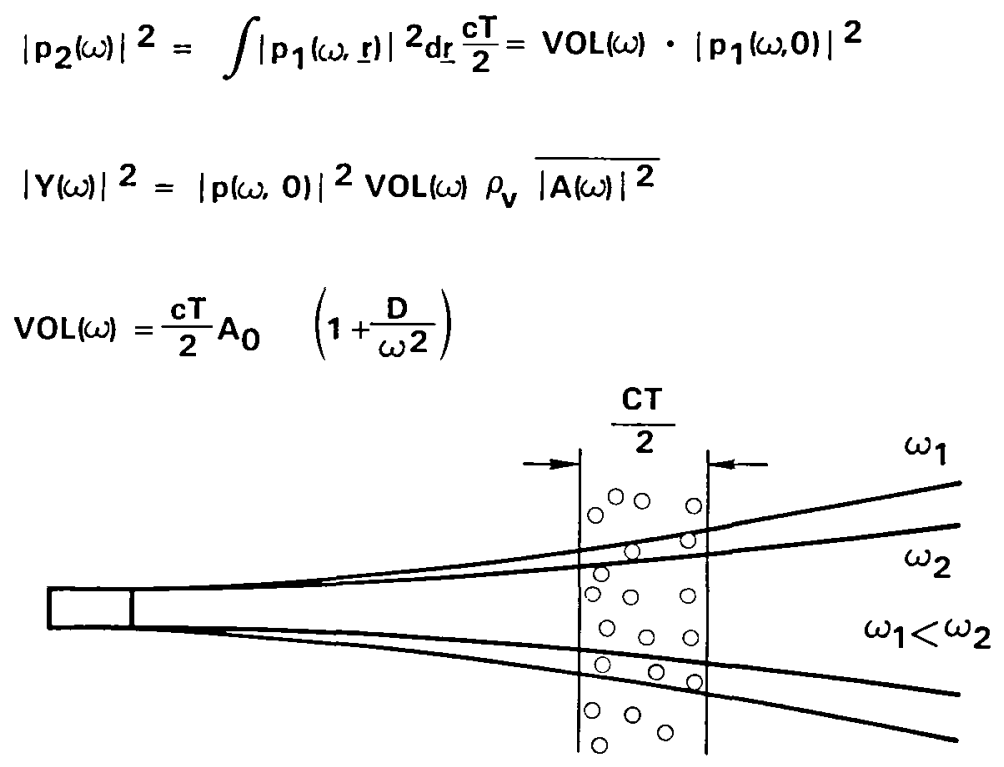

Figure 11 Diagram of pulse echo experiment measuring the backscatter from a region a distance $z_{1}$ from the transducer. 
The spectral density of $n(t)$ is found by taking the expectation of $n(\omega) n *(\omega)$ over the distribution of scatterer sizes and positions. If we assume that the scatterers are uniformly distributed in real space, that multiple scattering is negligible, and we restrict the frequency mesh to $\omega_{k}=2 \pi k / T$, we obtain

$$
E\left(n(\omega) n^{*}\left(\omega^{\prime}\right)\right)=\delta_{\omega \omega^{\prime}} \frac{c T}{2}\left(\iint_{-\infty}^{\infty}|p(\omega, \underline{r})|^{2} d^{2} \underline{r}\right) \rho_{v} E|A(\omega)|^{2},
$$

where $\rho_{\mathrm{v}}$ is the expected number of scatterers per unit volume, $\delta$, is the Kronecker delta, and $E|A(\omega)|^{2}$ is the expected squared scattering amplitude for a randomly chosen scatterer. For piston-source transducers, the integral of $|p(\omega, r)|^{2}$ is not analytically tractable. For Gaussian transducers, however, it is quite easy. In either case, we identify the "volume", vol $(\omega)$, of the beam, as a frequency dependent entity which may be defined by

$$
\operatorname{vol}(\omega)=(\mathrm{cT} / 2) \iint_{-\infty}^{\infty}|\mathrm{p}(\omega, \underline{\mathrm{r}})|^{2} \mathrm{~d}^{2} \underline{\mathrm{r}} /|\mathrm{p}(\omega, \mathrm{o})|^{2} .
$$

Fertig and Richardson [ref. 9] give approximate expressions for vol( $\omega)$ for which oblique incidence and curved surfaces are allowed. The term $p(\omega, 0)$ is the on-axis diffraction correction and may be determined from the work of Thompson and Grey [ref. 10, 11] for piston sources. These latter authors give explicit forms for $p(\omega, 0)$ which allow for the inclusion of a reference calibration wave, $R(\omega)$, which enables one to relate a pressure profile on the face of the transducer to a voltage reading. In the case in which the scatterers are spherical and for which the Rayleigh limit holds, one obtains

$$
\begin{aligned}
& E|n(\omega)|^{2} /|R(\omega)|^{2}= \\
& \left.\qquad \frac{2 C^{2}\left(z_{1}, z_{2}, \omega\right)}{k_{2} a^{2} D\left(z_{1}, z_{2}, \omega\right)}\right]^{2} \frac{1}{R_{1}^{2} R_{2}^{2}} \operatorname{vol}(\omega) \omega^{4} \eta \rho_{v} E\left(r^{6}\right)
\end{aligned}
$$

Here $C$ and $D$ are diffraction correction functions for the experiment and calibration, $R_{1}$ and $R_{2}$ are corner reflection coefficients associated with the calibration, $E\left(r^{6}\right)$ is the expected value of the sixth power of the pore radius, and $\eta$ is a frequency and size independent parameter related to the scattering of spheres in the host material. In the far fleld limit, the expression in brackets above is Independent of Erequency.

\section{COMPARISON BETWEEN THEORY AND EXPERIMENT}

The experimental approach centers around a broadband transducer which is used to launch a short pulse of shear wave energy into the material in many different directions as shown schematically for the spin-pit test sample in figure 12 . During its passage through the material, the microstructural inhomogeneities reflect energy back toward the transducer which is now used as a receiver to detect these small echoes which form a train of waveforms which have amplitudes and phase relationships depending on where and how strong the sources are. Several time traces recorded for a variety of regions for both samples $A$ and $C$ are shown in figure 13 . It is clear 


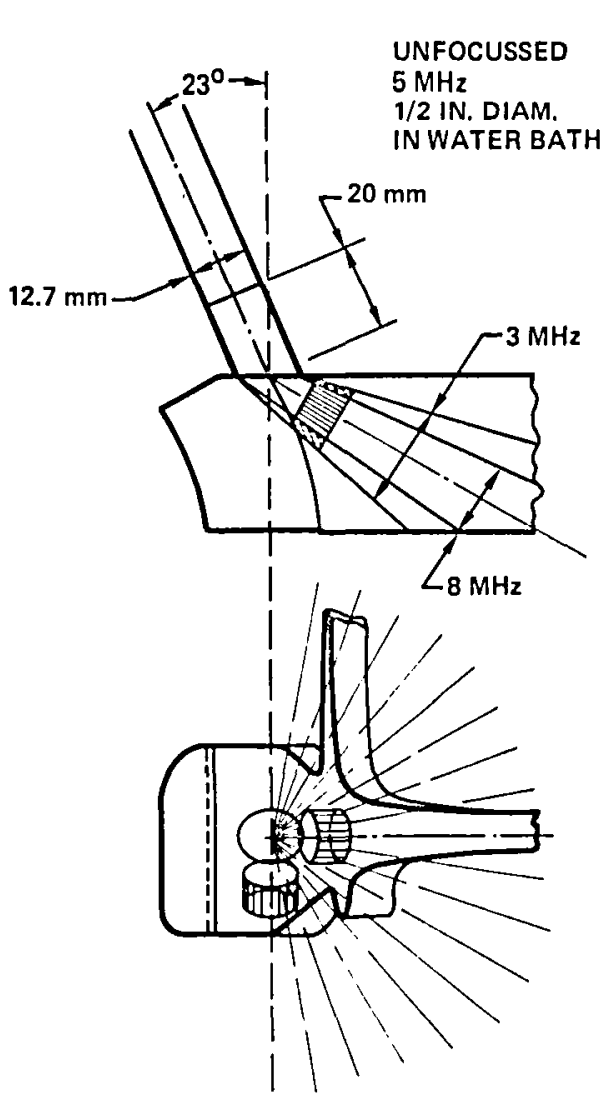

$\operatorname{SC83-23337}$

Figure 12 Schematic diagram of spin pit test specimen and ultrasonic shear wave beam.

that these signals are more or less random and form a background. In a typical run, the backscattered background signals are windowed at 10 different sections of the time trace corresponding to 10 different regions of the sample. Each waveform is time averaged over 512 transmission pulses to decrease electronic noise in the recelver equipment. The 10 waveforms are deconvolved with a reference signal to remove the transducer characteristics and averaged to produce one statistically representative waveform for the sample material from which now the power frequency spectrum may be calculated. Figure 14 shows the backscatter power spectra for samples $A$ and $C$ before deconvolution whereas figure 15 shows the same data after deconvolution and therefore presents the results in final form.

As Indicated by the dashed lines in figure 15 the predicted backscatter spectra have slopes which agree well with those obsrved, 1.e., a 3.25 power law for the frequency dependence of the backscattered intensity.

Another, more stringent, test of the data and model is to see whether a key microstructural feature may be predicted and compared with micrographical data. This is attempted in Table 3. Here the values of the estimated mean power densities for the two samples and the ultrasonic backscatter intensities at $10 \mathrm{MHz}$ are inserted into the model to calculate values of $\left\langle r^{6}\right\rangle^{1 / 6}$ which are then compared to corresponding values estimated from the micrographical examination. The comparisons are shown in the last two columns of the table. Considering that the micrographical examination was limited to the surfaces of the samples, the agreement is reasonable. The calculation demonstrated also that small errors in the count of the large pores introduces large errors in the final estimates. 


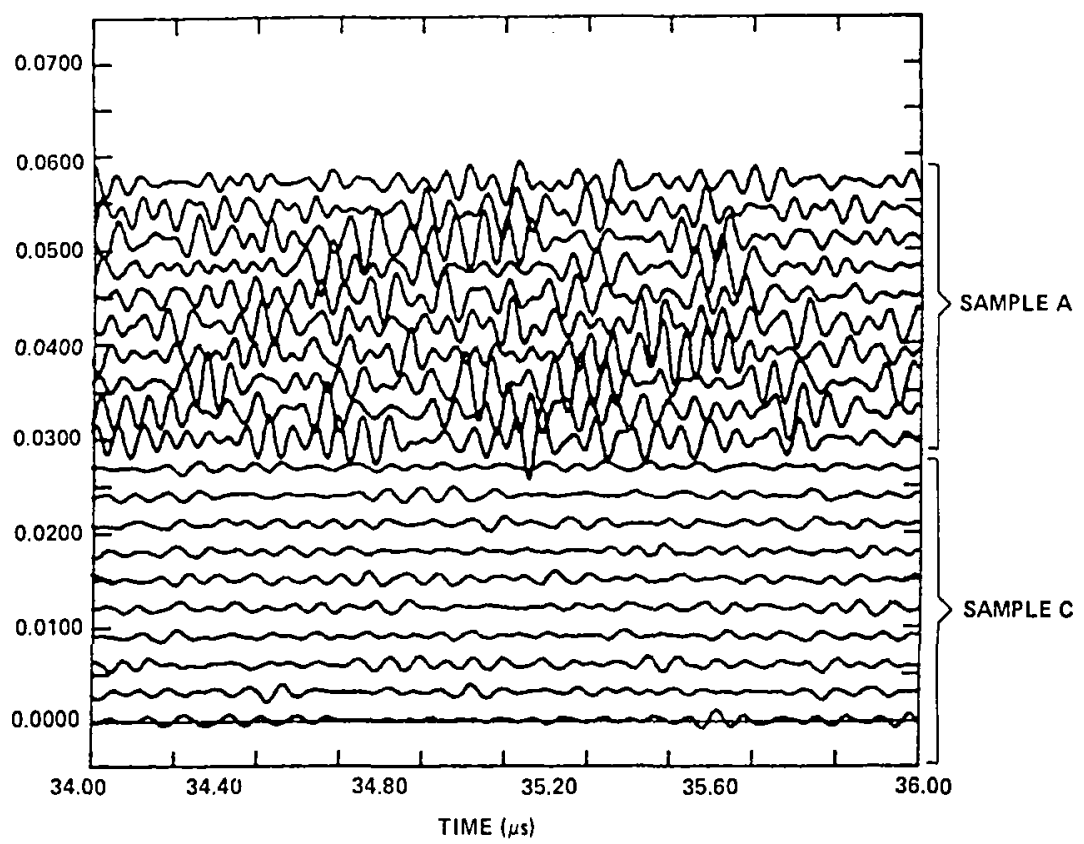

F1gure 13 U1trasonic backscattering noise in IN-100.

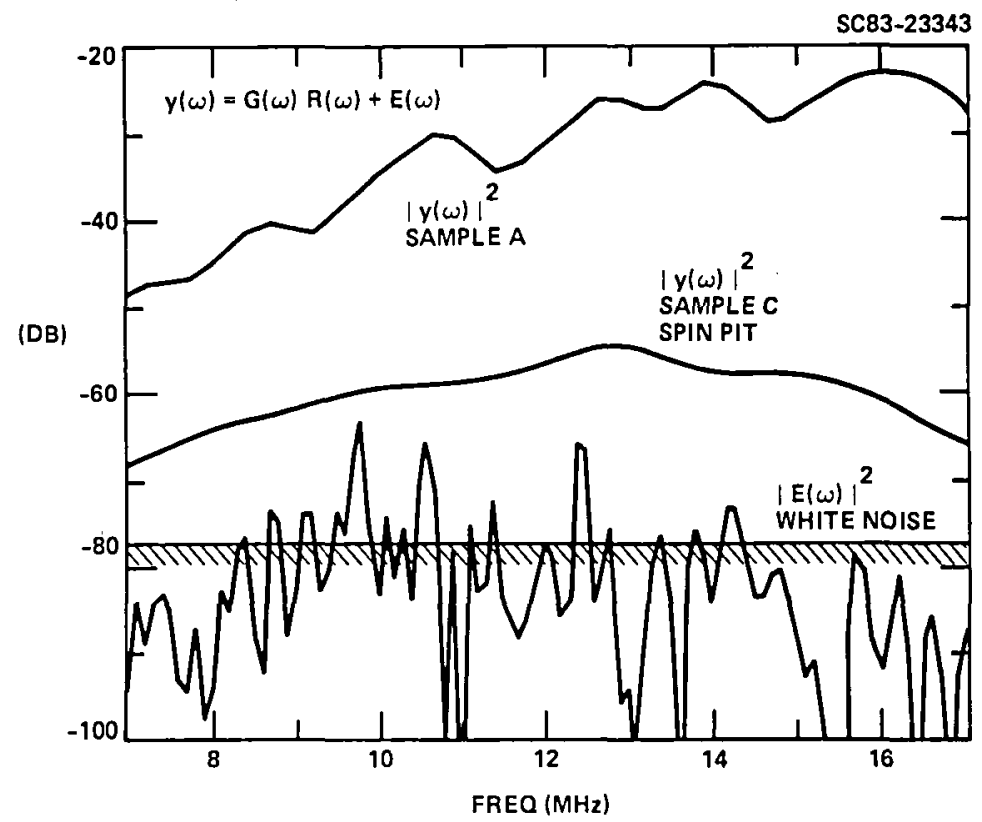

Figure 14 Ultrasonic backscatter power spectra for two samples of IN-100 before deconvolution. 


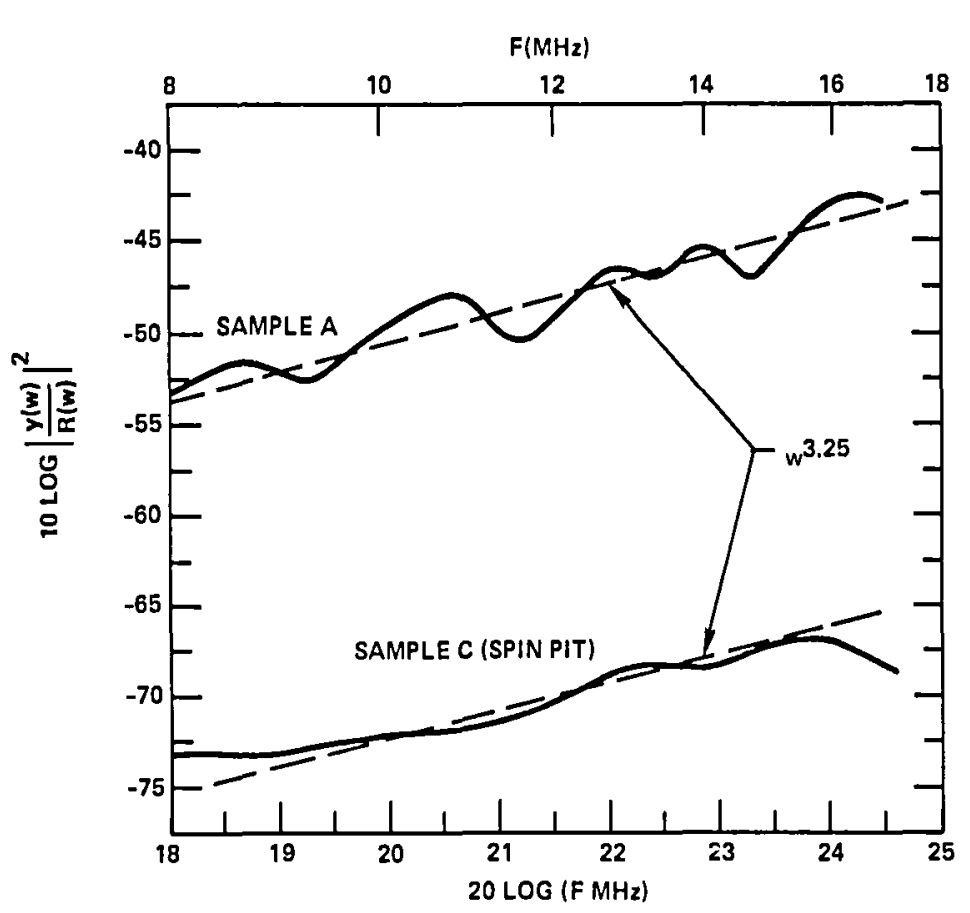

Figure 15 Ultrasonic backscattering spectra for two different samples of IN-100.

Table 3

\begin{tabular}{|c|c|c|c|c|c|}
\hline & $\begin{array}{l}\text { Pore Dens1ty } \\
\left(\mathrm{mm}^{-3}\right)\end{array}$ & $\begin{array}{c}\text { Pores in } \\
5 \mu \mathrm{s} \mathrm{Beam} \\
\text { Vol }-0.23 \mathrm{~cm}^{3}\end{array}$ & $\begin{array}{l}\left|\frac{Y}{R}\right|^{2} \\
\text { at } 10 \mathrm{MHz}\end{array}$ & $\begin{array}{l}\text { Scattering } \\
\langle\mathrm{r}\rangle \\
(\mu \mathrm{m})\end{array}$ & $\begin{array}{c}\text { Micrograph } \\
\langle r\rangle \\
(\mu \mathrm{m})\end{array}$ \\
\hline $\begin{array}{l}\text { Research } \\
\text { Sample A }\end{array}$ & 329 & $\begin{array}{l}80,000 \\
(-50 \mathrm{~dB})\end{array}$ & $1 \times 10^{-5}$ & 5.34 & $4.1-19.5$ \\
\hline $\begin{array}{l}\text { Spln P1t } \\
\text { Sample C }\end{array}$ & 89 & $\begin{array}{l}20,000 \\
\quad(-73 \mathrm{~dB})\end{array}$ & $5.01 \times 10^{-8}$ & 2.74 & $2.4-10.0$ \\
\hline
\end{tabular}

FORMAL APPROACH TO INVERSE PROBLEM

Whereas we have not attempted to solve the full inverse problem in this paper, it is appropriate to consider how one might attack this problem. For this purpose, it is convenient to formulate the problem in terms of a new set of random variables, called $v_{j i}$, which represent the number of spheres whose centers lie in a volume element $j k^{\prime}$ located at $\vec{r}_{j}$ in the material and who have radil in the range $\left(a_{k-1}, a_{k}\right)$ If we let $\hat{j}_{j}\left(a_{k}\right)$ represent the "counting" process equaling the actual number of spheres per unit volume with centers in $\delta V_{j}$ and radil less than or equal to $a_{k}$, we see

$$
v_{j k}=\delta V_{j}\left(N_{j}\left(a_{k}\right)-N_{j}\left(a_{k-1}\right)\right)
$$


Again, if we take the case of negligible multiple scattering, the Fourier transform of the time windowed measured waveform takes the form

$$
y(\omega)=\sum_{j k} \sum_{p}\left(\omega, \vec{x}_{j}\right) A\left(\omega, \tilde{a}_{k}\right) v_{j k}+\varepsilon
$$

where $\varepsilon$ is additive noise (e.g., electronic), and $A\left(\omega, \tilde{a}_{k}\right)$ is the scattering amplitude of a sphere with nominal radius $\tilde{a}_{k,} \varepsilon\left(a_{k-1}, a_{k}\right)$. The term $p\left(\omega, \vec{r}{ }_{j}\right)$ is the system response function for position $\vec{r}_{j}$, the "center of $\delta V_{j}$. The above formulation assumes that the $\delta V_{j}$ and $\delta a_{k}=a_{k}-a_{k-1}$ are all suitably small.

Contrary to the "usual" inversion problem wherein information on an isolated scatterer of interest is contained in the expected value of $y(\omega)$, for this case, the information on the statistics of the $v_{j k}$ is contained in the variance of $y(\omega)$.

We make the following assumptions for the observation time $T$,

$$
\begin{aligned}
& \text { 1. } p(\omega, \vec{r}) \doteq p(\omega, \underline{r}) e^{i \omega \tau}, \quad v=2 z / c . \\
& \text { 2. } v_{j k} \sim \text { stationary Polsson process } \\
& \text { 3. } \varepsilon(\omega) \sim \text { zero mean stationary process, independent of } v_{j k} \text {. }
\end{aligned}
$$

Then as $\delta \mathrm{V}_{j} \rightarrow 0, \Delta \mathrm{a}=\max \left|a_{k}-a_{k-1}\right| \rightarrow 0$, we have on the special frequency mesh $\omega_{\mathrm{k}}=2 \pi \mathrm{k} / \dot{\Phi}$,

$$
\begin{aligned}
& E\left(y\left(\omega_{k}\right)\right)=0 \\
& E\left(y\left(\omega_{k}\right) y *\left(\omega_{k},\right)\right)=\delta_{k k^{\prime}}\left[\frac{c T}{2} \int d^{2} \underline{r}\left|p\left(\omega_{k}, \underline{r}\right)\right|^{2}\right. \\
& \left.\cdot \int d a \rho(a)|A(\omega, a)|^{2}+C_{\varepsilon}(k) T\right],
\end{aligned}
$$

where $\delta_{\mathrm{kk}^{\prime}}$ is the Kronecker delta, $\rho(a)$ is the number density of spheres of radius $a$, and $C_{\varepsilon}(k) T$ is the variance of the $k^{\prime}$ th frequency component of the additive noise.

By inversion, we mean based on a set of samples of $y(\omega)$, estimate $p(a)$. Specifically, we are interested here in estimating the underlying statistics of the pore distribution, not the actual size and location of a specific set of pores in a particular region of the specimen. This latter question is, of course, of interest in many applications. Here, however, we are interested in the former. As is well known, this is typically an i11-posed problem. Large changes in $\rho(a)$ can result in small changes in $y(\omega)$. For that reason, it will probably be necessary to parameterize $\rho$. To that end, define $\rho_{3}$ as the number density of spheres and $\bar{F}_{3}(a ; \underline{\theta})$ as the fraction of spheres with radil greater than a. Thus 


$$
\rho_{3} \bar{F}_{3}(a ; \underline{\theta})=\int_{a}^{\infty} \rho(a) d a
$$

The subscript 3 is meant to reinforce the fact that we are estimating volume densitles of particles and not area densitles. The latter often forms the raw data from sectioning experiments. The inversion problem is to estimate $\rho_{3}$ and the parameter $\theta$ (possibly vector-valued). One approximation to use for estimation of $\rho 3$ and $\theta$ is to assume that $y(\omega)$ is a Gaussian process. This will be the case if the spheres are "dense" relative to the system response function so that no scatterer 1s "Isolated" in time. In the Gaussian case, the posterlor probability of p3 and $\theta$ given $y$ is

$$
\begin{aligned}
\ln P\left(\rho_{3}, \underline{\theta} \mid y\right)= & k-\frac{1}{2} \sum_{k} y^{*}\left(\omega_{k}\right) B_{k k} y\left(\omega_{k}\right) \\
& +\ln P\left(\rho_{3}, \underline{\theta}\right)+\frac{1}{2} \sum_{k} \ln B_{k k},
\end{aligned}
$$

where $K$ is a normalization constant, $P\left(\rho_{3}, \theta\right)$ is the prior distribution of $\rho_{3}$ and $\underline{\theta}$, and $B_{k k}$ is the inverse of the diagonal of the variance of $y\left(\omega_{k}\right)$. It is given by

$$
B_{k k}^{-1}=T C_{\varepsilon}\left(\omega_{k}\right)+M\left(\omega_{k}\right) \int \rho_{3} f_{3}(a ; \underline{\theta})\left|A\left(\omega_{k}, a\right)\right|^{2} d a,
$$

where

$$
M(\omega)=c T / 2 \int|p(\omega, \underline{r})|^{2} d^{2} \underline{r}
$$

and $f_{3}(a ; \underline{\theta})=-d \bar{F}_{3}(a ; \underline{\theta}) / d a$ is the probability density function of the sphere sizes.

Two classical sets of estimators present themselves. The first set is $\left\{\rho_{3}, \theta\right\}$ which maximizes the posterior density $P\left(\rho_{3}, \theta \mid y\right)$. These estimators define the mode of this distribution and represent the most probable values. The second set estimators is $\left\{\rho_{3}, \underline{\theta}\right\}$ defined by the means of marginals of $P\left(\rho_{3}, \underline{\theta} \mid y\right)$. Thus

$$
\begin{aligned}
& \hat{\rho}_{3}=\int \rho_{3} P\left(\rho_{3}, \underline{\theta} \mid y\right) d \rho_{3} d \underline{\theta} \\
& \hat{\hat{\theta}}=\int \underline{\theta} P\left(\rho_{3}, \underline{\theta} \mid y\right) d \rho_{3} d \underline{\theta}
\end{aligned}
$$

These estimators minimize the mean square error.

In either case, the posterior variance of the estimators will be reduced if multiple waveforms $y_{r}(\omega), r=1, \ldots, R$ are avallable. Having a small number of waveforms places a greater reliance on the prior density $P\left(\rho_{3}, \underline{\theta}\right)$ to keep the problem well-posed. 
The microstructure of a nickel-base powder metal alloy IN-100 has been studied with the aid of micrographical and ultrasonic techniques. The material showed grains of small diameter with a narrow distribution in the range of 2-4 $\mu \mathrm{m}$ and sparsely distributed micropores with diameter in the 5-20 $\mu \mathrm{m}$ range. The micropores dominated the ultrasonic attenuation and backscattering characteristics. The ultrasonic backscattering was selected to carry out quantitative measurements and calculations for comparison of theory and experiment. The frequency dependence from 8 to $18 \mathrm{MHz}$ of the backscattered signal was in good agreement with calculations which included the contributions from on-axis diffraction, back-surface reflection, the frequency dependent beam, the Rayleigh scattering and the distribution of scatterers. In addition to the forward problem, the inverse problem was addressed in two ways (1) by estimating the mean pore size $\left\langle\mathrm{r}^{6}\right\rangle^{1 / 6}$ from an approximate count of the pore density. This was found to give reasonably good agreement with the pore size estimated from micrographical information. (2) A formal approach for a stochastic Inversion was developed.

The results point the way toward the nondestructive detection and characterization of anomalous distributions of micropores where conventional ultrasonic imaging is difficult. Such information may be useful in the early detection of potential fallure sites where stress induced void coalescence leads to crack initiation.

\section{REFERENCES}

1. M. O'Donne11 and J.G. Miller, "Quantitative Broadband Ultrasonic Backscatter: An Approach to Nondestructive Evaluation in Acoustically Inhomogeneous Materials," J. Appl. Phys. 52, 1056 (1981).

2. K. Goebbels, "Method of Quantitatively Determining the Grain Size of Substances," United States Patent No. 4026,157, May 31, 1977.

3. E. M. Papadak1s, J. Acoust. Soc. A, 37, 711 (1965).

4. W. P. Mason and H. J. McSkimin, J. Appl. Phys. 19, 940 (1948).

5. I. M. Lifshitz and G. D. Parkhomovsk11, J. Expt. Theoret. Phys. (USSR) 20,175 (1950).

6. A. G. Evans, B. R. Tittmann, L. Ahlberg, B. T. Khun-Yakub, G. S. Kino, "Ultrasonic Attenuation in Ceramics," J. Appl. Phys. 49, 1669 (1978).

7. L. Adler, Proceedings of the Review of Progress in Quantitative NDE (edited by D.0. Thompson and D. Chiment1) Plenum Publishing Company, (1984).

8. B.R. Tittmann, L.A. Ahlberg and K. Fertig, Proceedings of the Review of Progress in Quantitative NDE (edited by D.0. Thompson and D. Chimenti) Plenum Publishing Company, 1983 and 1982.

9. K.W. Fertig and J.M. Richardson, "Computer Simulation of Probability of Detection," 1982 AF/DARPA Review of Quantitative NDE, Plenum Publishing Company. 
10. R.B. Thompson, "Diffraction Corrections for Scattering Measurement," Internal Report, Center of Advanced Nondestructive Evaluation, Ames Laboratory, Iowa State University (1982).

11. R. B. Thompson and T.A. Gray, "Analytical Diffraction Corrections in Ultrasonic Scattering Measurements," Proceedings of the Review of Progress In Quant1tative NDE, (edited by D.0. Thompson and D. Chiment1) Plenum Publish1ng Company, August 1-6 (1982). 